\title{
Dynamics carrier relaxation in InGaN/GaN Multiple Quantum Well Structures
}

\author{
Shih-Wei Feng ${ }^{a}$, Yung-Chen Cheng ${ }^{a}$, Yi-Yin Chung ${ }^{a}$, C. W. Liu ${ }^{a}$, Ming-Hua Mao ${ }^{a}$, Chih-Chung \\ Yang $^{1 \mathrm{a}}$, Yen-Sheng Lin ${ }^{\mathrm{b}}$, Kung-Jeng $\mathrm{Ma}^{\mathrm{b}}$, and Jen-Inn Chyi ${ }^{\mathrm{c}}$
}

(a) Department of Electrical Engineering and Graduate Institute of Electro-Optical Engineering, National Taiwan University, 1, Roosevelt Road, Sec. 4, Taipei, Taiwan, R.O.C.

(b) Department of Mechanical Engineering, Chung Cheng Institute of Technology, Tahsi, Taoyuan, Taiwan, R.O.C.

(c)Department of Electrical Engineering, National Central University, Taiwan, R.O.C.

Keywords: carrier dynamics, localized states, InGaN/GaN quantum well, carrier transport

\begin{abstract}
We report the fast and slow decay lifetimes of multi-component photoluminescence (PL) intensity decays in the time-resolved photoluminescence measurements at the room temperature and a low temperature (12K). The fast decay component was essentially due to carrier dynamics, that is, carrier transport from weakly localized to localized states. Such a carrier transport process results in extremely long PL decay time (up to almost $120 \mathrm{ns)}$ for strongly localized states at the low temperature. At room temperature, because of thermal energy and hence carrier escape from strongly localized states, effective lifetimes becomes shorter.
\end{abstract}

\section{Introduction}

Due to the low miscibility between $\mathrm{InN}$ and $\mathrm{GaN}$, indium aggregation and phase separation (formation of either InN or GaN clusters) occurred in InGaN/GaN quantum well (QW) structures ${ }^{1}$. Indium-rich clusters in such a $\mathrm{QW}$ structure form the localized states, which may trap carriers for radiative recombination ${ }^{2}$. Time-resolved photoluminescence (TRPL) results of InGaN/GaN QW samples have been reported by many groups ${ }^{3,4,5}$. However the decay mechanism in InGaN/GaN QWs has not been well discussed yet, particularly the non-single exponential decay feature ${ }^{4,6}$. In this paper, we report carrier dynamics of three InGaN/GaN QW samples with varied indium contents. We conducted TRPL measurements at room temperature (RT) and low temperature (LT) at $12 \mathrm{~K}$ with the same excitation photon energy. Different relaxation behaviors including two-component and one-component decays of PL intensity were observed. We discussed the mechanisms, which are responsible for the observed carrier dynamics.

\section{Sample Preparation and Experimental Procedures}

The samples were grown in a low-pressure metal-organic chemical vapor deposition reactor. The InGaN/GaN multiple QW samples consisted of five periods of Si-doped $\mathrm{InGaN}$ well with $3 \mathrm{~nm}$ in thickness. The designated indium compositions of the sample were 11,16 , and $21 \%$ (samples A, B, and C, respectively). The Si doping concentration was $10^{18} \mathrm{~cm}^{-3}$. The barrier was $7 \mathrm{~nm} \mathrm{GaN}$. In each sample, the QW layers were sandwiched with a $1.5 \mu \mathrm{m} \mathrm{GaN}$ bufferlayer on a sapphire substrate and a $50 \mathrm{~nm} \mathrm{GaN}$ cap layer. The growth temperatures were 1050 and $740{ }^{\circ} \mathrm{C}$ for $\mathrm{GaN}$ and InGaN, respectively.

The TRPL measurements were performed with a Hamamatsu streak camera (resolution $4.7 \mathrm{ps}$ ). Frequency-doubled optical pulses of about $100 \mathrm{fs}$ width were generated from a mode-locked Ti:sapphire laser for excitation. The pumping photon energy of TRPL was $3.1 \mathrm{eV}(400 \mathrm{~nm})$. The samples were placed in the cryostat system to measure the low temperature TRPL at $12 \mathrm{~K}$.

\section{Experimental Results and Discussions}

Figure 1 shows the temporal decay profiles of low temperature PL of sample B at different emission photon energy. The temporal behavior on the high-energy side shows a multiple-exponential decay with the fast and slow decays at the earlier

') Corresponding author; Phone: 886-23657624,Fax: 886-23652637, e-mail: ccy@cc.ee.ntu.edu.tw 
and later times, respectively. The fast-decay component tends to disappear when emission photon energy is below a certain value around $2.805 \mathrm{eV}$. For the multi-exponential decays, we fitted the fast and slow decays with individual single exponential for the fast and slow lifetimes. On the other hand, a single decay profile was fitted with a single exponential. Note that the stretched exponential model with the parameter $\beta[4,6]$ cannot fit well the early stage of the decay profiles on the high-energy side.

Fig. 2 shows the lifetimes of all the three samples at $12 \mathrm{~K}$. On the high-energy side, the fast decay time can be shorter than 300 ps. Such an order of magnitude of decay time can not be only attributed to radiative carrier recombination. In GaAs/AlGaAs multiple quantum-wells, the non-single exponential decay behavior was also observed ${ }^{7,8}$. It was attributed to carrier transfer between free-carriers and the bound states. In our system, besides recombination such a short decay time is due to an additional process, i.e., carrier transport from weak-localized states to strong-localized ones. Therefore, the fast-decay component corresponds to carrier recombination in weakly localized states as well as carrier transport from weakly localized states to strongly localized ones. Note that carrier dynamics includes carrier transport between different energy levels and different spatial locations. Such a process will disappear after a quasi-equilibrium state is reached. From Fig. 1, one can see that this carrier transport process lasts for several ns. The decreasing trends of the fast-decay lifetimes, as shown by the empty symbols in Fig. 2, are reasonable because it is easier for carriers in the weaker localized states to relax down to stronger localized states.

As for the slow-decay or long-term decay component, carrier capture of a strongly localized state occurs in a longer time range. This lifetime shows a peak in each of the three samples. For Sample C, this lifetime can be as larger as almost $120 \mathrm{~ns}$. Such a long lifetime is attributed to the quasi-balance of carrier decay due to recombination and net carrier supply due to carrier transport. Thus the decay slope could be considered to be the effective carrier lifetime for the interplay between carrier decay and supply. In each sample, the decreasing trend on the lower energy side of the lifetime peak can be attributed to the relatively higher difficulty for carrier to relax into the deeply localized states. This result may be related to state population distribution among localized states. At a low temperature, without thermal energy carrier relaxation into deeply localized states might be difficult.

To study the effects of thermal energy, we also conducted RT TRPL. Two RT TRPL intensity profiles of two samples at two photon emission energies are shown in Fig. 3. The two-component decay with clear turning point in either profile can be seen. We fitted our data with two-step exponential decay to obtain the fast decay time $\tau_{1}$ and slow decay time $\tau_{2}$. The slow decay time of the three samples are shown as functions of emission photon energy in the upper portion of Fig. 4 . The decay time $\tau_{2}$ increases with decreasing photon energy for each of all three samples. This is a typical property of the carrier localization model. The slow decay lifetimes can be regarded as mainly describing the recombination of localized excitons. Note that the comparison between Fig. 2 and the upper portion of Fig. 4 shows that the RT lifetimes are shorter than those of LT. This naturally results from the effect of defect recombination. Also, the monotonic variations of the RT results can be attributed to the fact that at RT it is easier to reach a quasi-equilibrium condition among all localized states because of the thermal energy.

For the fast decay part, $\tau_{l}$ on the high-energy side describes the decay rate of the combined effect of 1) carrier transport from weakly localized states to strongly localized states; 2) non-radiative recombination related to defects; 3 ) radiative recombination of carriers in weakly localized states. On the other hand, $\tau_{l}$ on the low-energy side describes the decay rate of the combined effect of 1) carrier escape from strongly localized states to weakly localized ones and defects via thermal energy; 2) radiative recombination. Note that carrier escape means the net effect of carrier capture and carrier go-away. With the thermal energy, carrier escape results in insignificant increasing trend of the fast decay time with decreasing photon energy. This is a difference between RT and LT results, as shown by the comparison between Fig. 2 and the lower portion of Fig. 4.

\section{Conclusions}

In summary, we have demonstrated the fast and slow decay times of multi-component PL intensity decay in the TRPL measurements at both RT and LT. The fast decay was essentially due to carrier dynamics between strongly localized and weakly localized states. At room temperature, due to thermal energy and hence carrier escape from strongly localized states, effective lifetimes becomes shorter.

\section{Acknowledgements:}

This research was supported by National Science Council, The Republic of China, under the grants of NSC 90-2112-M-002-052, NSC 90-2215-E-002-027, and NSC 90-2215-E-002-041, also by Chung-Shan Institute of Science and 
Technology.

\section{References}

[1] Y. S. Lin, K. J. Ma, C. Hsu, S. W. Feng, Y. C. Cheng, C. C. Liao, C. C. Yang, C. C. Chou, C. M. Lee, and J. I. Chyi, Appl. Phys. Lett. 77, 2988 (2000).

[2] T. Hino, S. Tomiya, T. Miyajima, K. Yanashima, S. Hashimoto, and M. Ikeda, Appl. Phys. Lett. 76, 3421 (2000).

[3] H. S. Kim, R. A. Mair, J. Li, J. Y. Lin, and H. X. Jiang, Appl. Phys. Lett. 76, 1252 (2000).

[4] M. Pophristic, F. H. Long, C. Tran, I. T. Ferguson, and R. F. Karlicek, Jr., J. Appl. Phys. 86, 1114.

[5] Y. Narukawa, Y. Kawakami, Sz. Fujita, Sg. Fujita and S. Nakamura, Phys. Rev. B 55, R1938 (1997).

[6] J. -Chr. Holst, A. Hoffmann, D. Rudloff, F. Bertram, T. Riemann, J. Christen, T. Fery, D. J. As, D. Schikora, and K. Lischka: Appl. Phys. Lett. 76, 2832 (2000).

[7] H. X. Jiang, E. X. Ping, P. Zhou, and J. Y. Lin, Phys. Rev. B 41,12949 (1990).

[8] M. Kohl, D. Heitmann, W.W. Rühle, P. Grambow, and K. Ploog, Phys. Rev. B 41,12338 (1990).

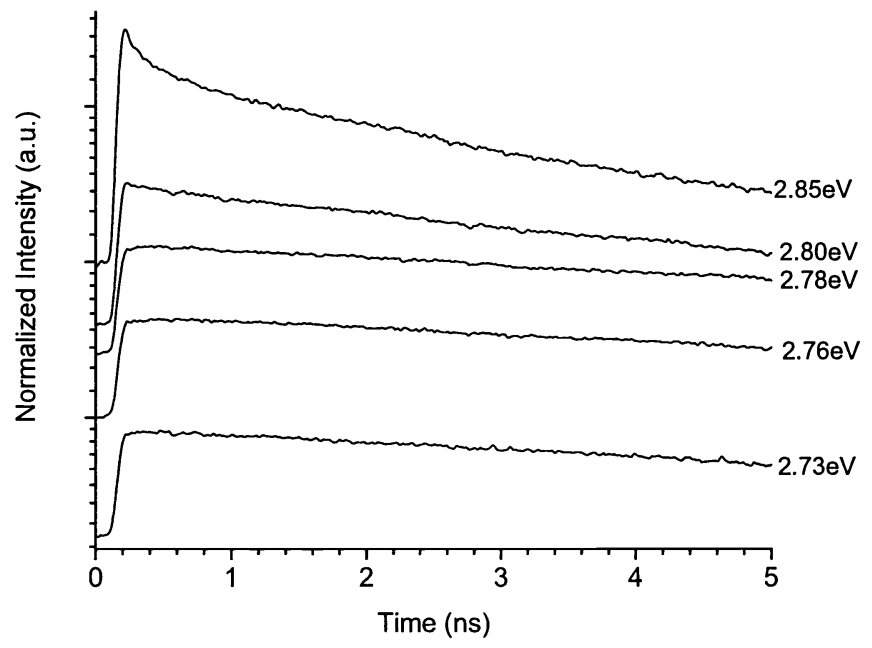

Fig. 1 Temporal decay profiles of low temperature (12K) PL of sample B at different emission energy.

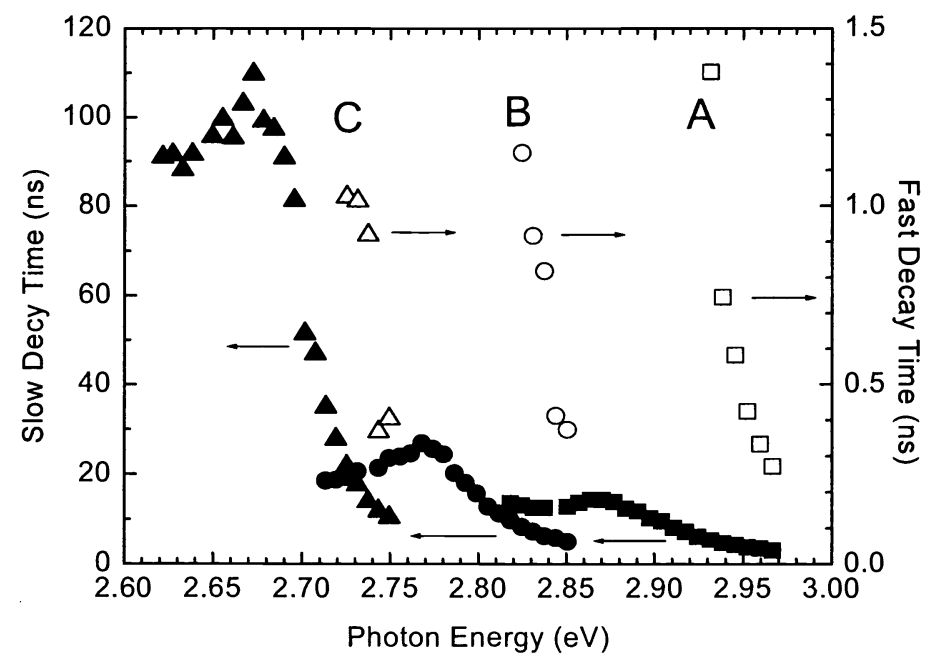

Fig. 2 Decay time of fast and slow components as functions of emission photon energy of all three samples at 12K. 


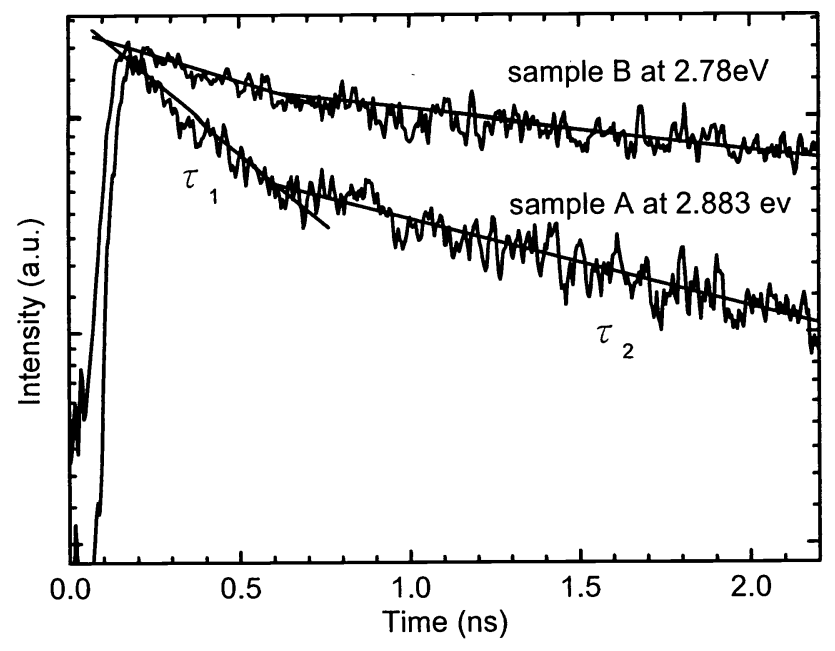

Fig. 3 Typical TRPL profiles and fitting curves of sample A and B at room temperature.

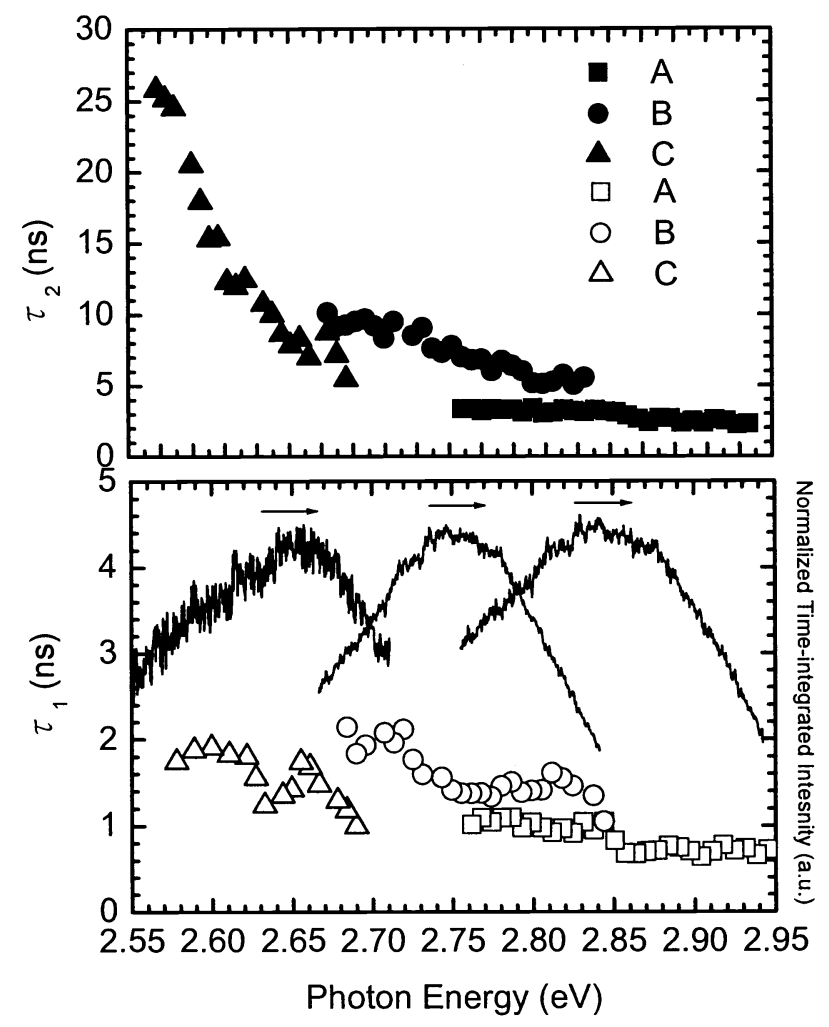

Fig 4. Decay time $\tau_{1}$ and $\tau_{2}$ as functions of emission photon energy for the three samples at RT. 